\title{
Análise Espacial da Eficiência dos Gastos Públicos em Saúde em Minas Gerais
}

\section{Efficiency of Public Health in Minas Gerais State: a Spatial Analysis}

\author{
Thiago Costa Soares* \\ Jenniffer Beatriz da Costa** \\ Luckas Sabioni Lopes***
}

Resumo: Esta pesquisa analisa a relação espacial da eficiência municipal em saúde em Minas Gerais, tomando como referência os 853 municípios do estado em 2010. Para criar os indicadores de eficiência, utiliza-se a técnica de análise envoltória de dados meta-frontier. O estudo espacial é realizado por meio da análise exploratória de dados espaciais. Os principais resultados destacam problemas na aplicação dos recursos e elevado montante relativo de gastos, sobretudo nos pequenos municípios. Pela análise espacial, encontram-se aglomerados de alta e baixa eficiência no estado. Ao se comparar os agrupamentos, verifica-se que os municípios "alto-alto" apresentam indicadores socioeconômicos mais elevados em relação aos "baixo-baixo”. Estudar melhor a realidade dessas regiões poderia ser o primeiro passo para otimizar os recursos aplicados na saúde.

Palavras-chave: Eficiência. Saúde. Análise espacial.

Abstract: In this paper, we analyzed the spatial dependence of the efficiency of publicsector health services in Minas Gerais, taking as reference its 853 municipalities in 2010. We computed efficiency scores and rakings by employing the method of data envelopment analysis meta-frontier. Spatial analysis was carried through with exploratory data techniques. Our results highlighted problems with resource allocation, especially in smaller cities. In addition, we found clusters of high and low-efficiency scores in the state. When comparing groups, it was verified that municipalities classified as "high-high" had higher socioeconomic indicators than those in the "low-low" regions. Therefore, a proper understanding of local realities could represent a step toward the optimization of resource allocation in the public health system.

Keywords: Efficiency. Public health. Spatial analysis.

JEL Classification: C02; I18; R01.

* Doutor em Economia Aplicada pela Universidade Federal de Viçosa (UFV). Professor adjunto do Departamento de Economia da Universidade Federal de Juiz de Fora, campus Governador Valadares (UFJF). E-mail: thiago.costa@ufjf.edu.br

* Graduada em Economia pela Universidade Federal de Juiz de Fora, campus Governador Valadares (UFJF). E-mail: jenniffer-beatriz@hotmail.com

*** Doutor em Economia Aplicada pela Universidade Federal de Viçosa (UFV). Professor adjunto do Departamento de Economia da Universidade Federal de Juiz de Fora, campus Governador Valadares (UFJF). E-mail: luckas.lopes@ufjf.edu.br 


\section{1 lntrodução}

A universalização do sistema de saúde no Brasil por meio da ação do Estado tem como marco a Constituição Federal (CF) de 1988. Pelo artigo no 196, a CF estabelece que "a saúde é direito de todos e dever do Estado, garantido mediante políticas sociais e econômicas que visem à redução do risco de doença e de outros agravos e ao acesso universal e igualitário às ações e serviços para sua promoção, proteção e recuperação" (BRASIL, 1988, não paginado). Para esse fim, criou-se o Sistema Único de Saúde (SUS), cuja abrangência, em teoria, deveria integrar e difundir gratuitamente o acesso aos serviços prestados.

Entre 1990 e 2015, o investimento per capita na saúde, em termos reais, mais que dobrou, tendo os indicadores de expectativa de vida e mortalidade infantil melhorado de forma expressiva (WORLD BANK, 2016). ${ }^{1}$ Contudo, o Brasil ainda enfrenta desafios, como a ineficiência na aplicação de recursos e os problemas de equidade e qualidade na provisão de serviços (MEDICI, 2011).

As particularidades do sistema brasileiro de saúde motivaram diversos estudos direcionados à questão da eficiência do gasto público nessa área. Nesse contexto, a literatura sobre eficiência vem se consolidando na avaliação da gestão dos serviços oferecidos pela esfera pública, pois permite comparar unidades e processos, delineando caminhos de convergência para que os recursos sejam alocados de forma ótima. É por essa, entre outras razões, que a construção de indicadores de eficiência se tornou caminho natural para o aprimoramento de políticas públicas, principalmente em países como o Brasil, que possuem elevada participação do Estado no setor (FARIA; JANNUZZI; SILVA, 2008; FONSECA; FERREIRA, 2009; MACHADO JÚNIOR; IRFFI; BENEGAS, 2011; SILVA et al., 2012).

Para citar alguns exemplos, Ferreira e Pitta (2008), estudando municípios paulistas, constroem indicadores de eficiência e concluem que mais da metade da amostra foi ineficiente. Em um trabalho semelhante aplicado a municípios fluminenses, Faria, Jannuzzi e Silva (2008) revelam baixos índices de eficiência. Analisando a situação das microrregiões de Minas Gerais, Fonseca e Ferreira (2009) mostram que cerca de 78\% estão em situação intermediária ou ruim na gestão.

Por outro lado, os estudos citados, como diversos outros, condicionam a análise de eficiência à ideia de que todos os municípios ofertam os mesmos serviços de saúde. Tal situação pode não representar a realidade dos municípios no Brasil, pois, conforme argumentam Rocha et al. (2017), a divisão dos recursos introduz heterogeneidade na provisão de serviços, a qual é geralmente definida por critérios populacionais (escala). Esse fator, se não controlado, pode causar erros na definição do modelo e na medição da eficiência.

$1 \quad$ Nesse período, a expectativa de vida subiu de 66 para 75 anos e a taxa de mortalidade infantil caiu de 52 para 14 óbitos para cada 1.000 nascidos vivos (WORLD BANK, 2016). 
Tratando-se ainda da gestão, outro ponto pouco explorado em estudos aplicados ao Brasil é a possível relação espacial entre os indicadores de eficiência em saúde. Felder e Tauchmann (2013) argumentam que o desempenho na gestão do sistema de saúde pode estar sujeito à dependência espacial em razão da migração inter-regional de pacientes e dos possíveis processos de aprendizagem. Os padrões regionais de eficiência, caso existam, podem apontar ainda bons modelos regionais de saúde e indicar localidades que necessitam de maior atenção.

Diante do exposto, o presente estudo pretende oferecer um panorama adicional à análise de eficiência da gestão pública em saúde, construindo e relacionando espacialmente indicadores de eficiência. Para isso, utilizam-se dados dos municípios de Minas Gerais de 2010. Esse estado torna-se interessante nesse cenário, uma vez que sua política pública visa organizar claramente os arranjos produtivos, concentrando determinados serviços e dispersando outros que se beneficiam menos de economias de escala (MARQUES et al., 2009). A relação espacial da eficiência municipal em saúde, dessa forma, pode representar padrões de gestão no âmbito regional, análise ainda não explorada pela literatura prévia.

Metodologicamente, utilizou-se a análise envoltória de dados (DEA) metafrontier, método não paramétrico que permite construir escores de eficiência dos gastos públicos em saúde considerando a heterogeneidade da amostra de municípios. Em seguida, aplicou-se a análise exploratória de dados espaciais (Aede), especificamente as estatísticas I de Moran global e I de Moran local, que permitem investigar a relação espacial dos indicadores de eficiência.

Este estudo está organizado em mais cinco seções, além desta introdução: a segunda apresenta uma revisão dos artigos sobre eficiência na saúde no Brasil; a terceira define o modelo teórico que embasa a construção de fronteiras de eficiência em saúde; a quarta descreve a metodologia e as variáveis; a quinta discute os resultados; e, por fim, a sexta apresenta as considerações finais do estudo.

\section{Revisão de Literatura}

É possível encontrar na literatura nacional diversos trabalhos que analisam a eficiência dos gastos públicos em saúde com o método DEA. Fonseca e Ferreira (2009) investigam os níveis de eficiência em unidades institucionais de saúde, tomando como referência as microrregiões de Minas Gerais, com retornos variáveis e orientação produto. Os autores utilizam estabelecimentos, equipamentos e profissionais como insumos e famílias acompanhadas e produção ambulatorial como produtos. Os resultados apontam a existência de lacunas gerenciais que podem ser supridas, segundo os autores, por políticas públicas com intervenções qualitativas e quantitativas in loco. No entanto, ressalta-se que as microrregiões de Minas Gerais não são esferas deliberativas de recursos públicos. Portanto, análises de 
eficiência nessa instância não necessariamente apontam caminhos para melhorar a alocação de recursos a nível municipal.

Machado Júnior, Irffi e Benegas (2011) verificam a eficiência dos gastos públicos com educação, assistência social e saúde nos municípios cearenses em 2005, utilizando quatro modelos DEA com orientação insumo e retornos constantes de escala. Seus resultados mostram baixa eficiência técnica dos gastos nesses setores. Nessa pesquisa, os autores não admitem outras possibilidades de retornos de escala (crescentes e decrescentes), configurando uma limitação em suas análises (MEDICI, 2011). Conforme destaca Medici (2011), a eficiência na aplicação dos recursos em saúde se relaciona com a fase produtiva (escala produtiva) dos municípios. Por exemplo, grandes centros normalmente operam com retornos decrescentes de escala. Já as menores localidades tendem a produzir com retornos crescentes.

Silva et al. (2012) avaliam a eficiência na alocação dos recursos destinados à educação, saúde e habitação em 404 municípios mineiros (47\% do total). No setor de saúde, eles adotam as variáveis gasto per capita e PIB per capita como insumos. As variáveis produto definidas foram o percentual da população atendida por programas de saúde da família (PSF) e cobertura vacinal média (poliomielite, tetravalente, hepatite $\mathrm{B}$ e febre amarela em menores de um ano, tríplice viral na população com um ano e influenza em maiores de 60 anos). A pesquisa conclui que diversas localidades não ofertam adequadamente serviços de saúde. Nesse trabalho, também se destaca o número elevado de missing data.

A pesquisa de Silva et al. (2012) permite levantar um questionamento importante sobre a escolha da variável PIB como recurso nessa função de produção. Por construção, cada município decide como alocar seus recursos, de modo a maximizar sua produção em saúde. Não obstante, o PIB não faz parte do conjunto de escolhas da gestão pública municipal. Isto é, tecnicamente, essa variável pode não ser apropriada na construção de indicadores de eficiência do setor, pois a gestão municipal não tem controle direto sobre ela. Ademais, em modelos com orientação input, a introdução desse fator forneceria análises incompatíveis com a realidade, uma vez que a função objetivo dos municípios minimiza a quantidade de recursos adotados (incluindo o PIB).

Queiroz et al. (2013) pesquisam a eficiência dos gastos em saúde em municípios do Rio Grande do Norte. Cobertura vacinal, atendimentos pelo PSF, procedimentos ambulatoriais e número de leitos foram as variáveis produto escolhidas. Como variável insumo, utilizou-se o gasto público total. Os pesquisadores encontraram evidências de ineficiência nesse estado, sobretudo em municípios menos populosos. No entanto, o número elevado de regiões excluídas da amostra pode ter prejudicado as estimativas da eficiência nessa pesquisa. 
Mazon, Mascarenhas e Dallabrida (2015), por seu turno, estudam a eficiência da gestão dos recursos do SUS em sete municípios do estado de Santa Catarina. As localidades escolhidas pertencem a regiões com baixos índices de desempenho da esfera pública. Nessa amostra, três localidades obtiveram indicadores superiores a $90 \%$, sendo somente uma eficiente. Um ponto crítico nesse trabalho foi a construção de indicadores de eficiência com um número pouco expressivo de municípios. Nesse contexto, os escores de eficiência obtidos podem não representar adequadamente a realidade do estado analisado.

Em relação ao método empregado, os estudos citados se embasam na premissa de homogeneidade da estrutura tecnológica. Em termos práticos, isso significa que os serviços em saúde ofertados por cidades grandes e pequenas são compatíveis e comparáveis. Porém, essa condição não se mantém em avaliações da gestão dos recursos públicos em saúde, pois a divisão das rubricas financeiras, os equipamentos e a natureza dos serviços prestados são baseados em critérios variados, como posição geográfica, tamanho populacional, possibilidades de economias de escala, etc. (MEDICI, 2011). Em outras palavras, seria mais adequado comparar municípios com estruturas produtivas similares (O'DONNELL et al., 2008).

No Brasil, essa problemática é discutida em Rocha et al. (2017). Os autores estudaram os gastos municipais em saúde no Brasil, adequando a análise à importância do tamanho populacional na definição da oferta dos serviços. Rocha et al. (2017) argumentam que a divisão dos recursos baseada em critérios populacionais introduz heterogeneidade na amostra, causando erros na definição do modelo DEA. Basicamente, a estratégia empírica consiste na subdivisão prévia dos municípios, formando grupos com estruturas de saúde mais homogêneas (O'DONNELL; RAO; BATTESE, 2008).

Além desse ponto, Felder e Tauchmann (2013) chamam a atenção para a possível relação espacial na gestão dos serviços em saúde. Em uma amostra composta por unidades de saúde na Alemanha, os autores constatam que as eficiências das unidades podem estar espacialmente correlacionadas, hipótese particularmente interessante para identificar padrões de eficiência. A questão espacial, no entanto, ainda não foi abordada em estudos nacionais.

Em resumo, é possível perceber que o DEA tem sido amplamente empregado em análises da eficiência em saúde. Um dos motivos é que a técnica permite comparar práticas de gestão e definir um rol de unidades que gerenciam os recursos de forma mais adequada. Por outro lado, a discussão sobre os problemas metodológicos do DEA na presença de heterogeneidade amostral (caso municipal) e a possivel correlação espacial da eficiência ainda são incipientes.

Dessa forma, embora haja vasta literatura que aborde a eficiência do gasto em saúde em diversas regiões do Brasil, este estudo pretende preencher uma lacuna, construindo um modelo DEA mais adequado à heterogeneidade dos dados 
e analisando a possível dependência espacial da eficiência da gestão pública em saúde no estado de Minas Gerais.

\section{A Função de Produção dos Serviços de Saúde}

Os serviços de saúde podem ser representados por uma função de produção municipal que especifica a produção em relação aos recursos empregados no setor. Por essa configuração, os estudos que analisam a eficiência focalizam em pressupostos microeconômicos, especificamente na fronteira de possibilidades de produção (FPP). Esta é uma representação de curto prazo de um processo produtivo que une determinados insumos a seus respectivos produtos. No caso da saúde, os insumos são a quantidade de máquinas, veículos, número de funcionários e/ ou quaisquer outros fatores aplicados que contribuam para a produção de serviços de saúde. Porém, dada a dificuldade em mensurá-los em análises empíricas, sobretudo em pequenas regiões, normalmente são empregados os gastos financeiros como proxies. Como produtos, podem-se citar número de vacinas, indicadores de qualidade, entre outros. Formalmente, escreve-se serviços $=\mathrm{f}$ (gastos).

Seguindo-se a relação estabelecida pela FPP, espera-se que a resposta da produção seja positiva quando os recursos se elevam. No entanto, a expansão dos serviços de saúde pode não ser proporcional, uma vez que a relação insumoproduto depende do fator escala (SANTERRE; NEUN, 2012). Ocorre que o custo médio em pequenas regiões é geralmente decrescente. Nesse estágio de produção, o aumento da oferta é mais que proporcional aos recursos aplicados, posto que se reduz a importância dos custos fixos no processo de produção, como aluguéis, salários dos servidores, entre outros (os quais não diminuem com a redução da produção). Por outro lado, em regiões maiores, o custo variável médio pode dominar o custo médio total, expandindo-se mais que proporcionalmente para cada serviço prestado. Nessas regiões, espera-se observar elevação menos que proporcional dos serviços em relação aos recursos empregados. Por meio de uma representação gráfica, pode-se visualizar melhor uma função de produção estática de curto prazo aplicada à saúde na Figura 1. 
Figura 1 - Função de produção de curto prazo na saúde

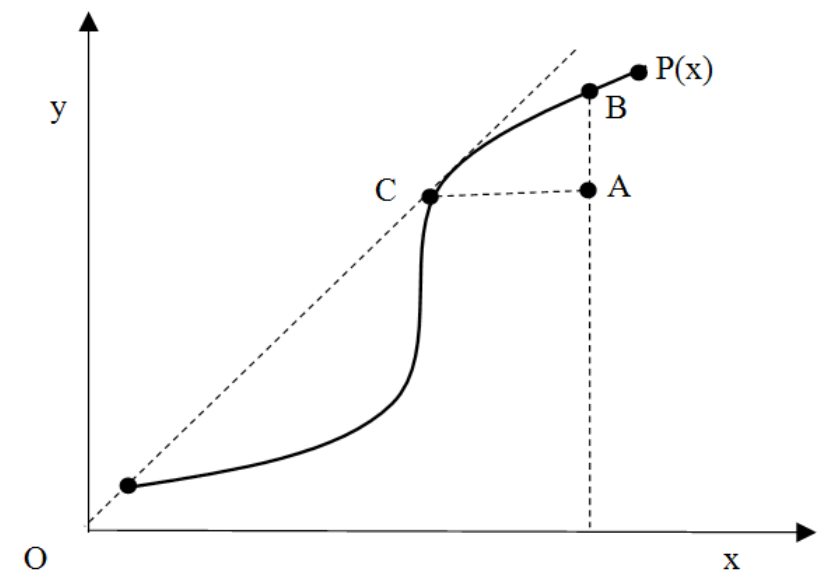

Fonte: Elaboração própria.

$y$ e $x$ variáveis que representam a produção e o gasto em saúde, respectivamente. A retilínea pontilhada permite visualizar os estágios de produção com retornos crescentes (distância $O C$ ), constantes (sobre $C$ ) e decrescentes (ponto $C$ em diante). Os municípios que se encontram sobre a fronteira $P(x)$ são tecnicamente eficientes, pois não há melhores práticas para serem comparadas (pontos $C$ e $B$ ). Pode-se atribuir indicadores unitários para representar a eficiência nesses pontos. Para os demais, ainda há algum caminho a percorrer até a fronteira, de modo que parte dos recursos ainda possa ser poupada para o mesmo nível produtivo, ou, de outra forma, ainda seja possível expandir a produção, mantendo-se o gasto fixo (ponto A). A distância até a fronteira representa o grau de eficiência (mensurado por um indicador menor que a unidade).

Na Figura 1, embora o ponto $A$ seja ineficiente, há dois vetores factíveis que podem projetá-lo para a fronteira. O primeiro é deslocando-o para o ponto $C$, reduzindo o insumo (recursos), mas fixando a produção (serviços de saúde). Essa escolha é denominada "orientação insumo - input", visto que o movimento de sentido à fronteira é feito em $x$. O escore de $A(\theta)$ é dado por $0<\frac{y_{A} / x_{A}}{y_{C} / x_{C}}=\theta<1$, resultando um vetor de projeção $A C$. O inverso de $\theta$ é conhecido como distância de Shepard (1970).

O segundo vetor é obtido pelo deslocamento de $A$ até $B(A B)$. Percebe-se que, nesse momento, mantém-se o insumo (recurso) de $A$, projetando sua produção (serviços de saúde) para $B$. Essa escolha é denominada "orientação produto - output”. O escore de eficiência ( $\phi$ ) é obtido por $0<\frac{y_{A} / x_{A}}{y_{B} / x_{B}}=\phi<1$. 
Dessa forma, pela perspectiva input, eficiência na saúde é a habilidade para produzir serviços de saúde ao menor custo possível, dadas as restrições tecnológicas. Na output, trata-se da máxima produção, fixando-se o gasto público. Generalizando, o ponto ótimo de Pareto, segundo Koopmans (1951), é alcançado quando não for possível aumentar a quantidade de serviços, para dado vetor de insumos, sem que outro serviço seja reduzido, ou reduzir os insumos, sem que isso gere redução da produção ou aumento de outro insumo. Pode-se dizer que eficiência técnica na saúde é a comparação relativa entre a razão $(y / x)$ realizada com a razão $(y / x)$ * ótima (FÄRE et al., 2005). Essa definição pode ser estendida para se lidar com a produção de diversos serviços de saúde.

\section{Metodologia}

A análise espacial da eficiência da gestão municipal em saúde em Minas Gerais foi realizada por meio da técnica Aede. Os índices de eficiência, por seu turno, foram construídos aplicando-se o método DEA. Os detalhes dos procedimentos metodológicos utilizados na pesquisa são descritos nas próximas três subseções.

\subsection{A Construção dos Indicadores de Eficiência em Saúde}

Na presente pesquisa, adotou-se a metodologia DEA, com orientação insumo, para se estimar os escores de eficiência dos gastos em saúde. O DEA é uma técnica não paramétrica que permite a construção de FPPs através de programação matemática. Esse método tornou-se uma das principais ferramentas de avaliação de desempenho dos municípios na gestão dos recursos destinados à saúde, pois, além de não necessitar de informações sobre o sistema de precificação, flexibiliza a forma funcional da FPP (FARIA; JANNUZZI; SILVA, 2008; FERREIRA; PITTA, 2008; FONSECA; FERREIRA, 2009). Consideram-se vetores de insumos $x_{i n}=\left(x_{1 n}, \ldots, \mathrm{x}_{l n}\right) \in \mathfrak{R}_{+}^{I}$ e produtos $y_{d n}=\left(y_{1 n}, \ldots, y_{D n}\right) \in \mathfrak{R}_{+}^{D}$ para toda unidade tomadora de decisão (decision making units - DMU) $\left(n \in \mathfrak{R}_{+}^{N}\right)$, conforme a expressão 1:

$$
\mathrm{P}(\mathrm{x})=\{(\mathrm{y}, \mathrm{x}): \mathrm{x} \operatorname{produz}(\mathrm{y})\},
$$

em que $P(x)$ representa a produção municipal de serviços de saúde para dado nível de recursos. Os municípios eficientes são aqueles que se posicionam sobre a FPP no ponto onde não é possível reduzir os gastos sem retrair a produção. A ineficiência na aplicação de recursos é medida pela distância em relação à fronteira.

A construção do DEA normalmente assume que as unidades produzem com a mesma tecnologia. Por outro lado, percebe-se que essa condição pode ser vio- 
lada em uma amostra composta por municípios, pois fatores como localização geográfica, condições socioeconômicas e tamanho populacional interferem na forma em que os recursos são otimizados. Por questões de escala, por exemplo, cidades grandes tendem a concentrar serviços de saúde mais complexos. Por isso, a comparação direta de estruturas produtivas diferentes pode causar problemas teóricos e empíricos.

Este estudo pretende dar maior atenção a esse ponto, utilizando, para isso, a análise meta-frontier. Em resumo, entende-se que os problemas causados pela heterogeneidade tecnológica podem ser reduzidos se os municípios forem divididos em grupos mais homogêneos. Essa é a ideia básica envolvida no procedimento sugerido por O’Donnell, Rao e Battese (2008). Os autores propõem construir indicadores de eficiência em um conjunto metafronteira dado por $\mathrm{T}^{\mathrm{m}}=\mathrm{P}(\mathrm{x})^{m}=\{(\mathrm{x}, \mathrm{y}): \mathrm{x}$ produz $(\mathrm{y})\}$, considerando toda a amostra disponível, isto é, supondo a existência de apenas uma fronteira tecnológica. Em seguida, supõem a existência de $k$ grupos formados por unidades com tecnologias semelhantes, de forma que $\mathrm{T}^{k}=\mathrm{P}(\mathrm{x})^{k}=\{(\mathrm{x}, \mathrm{y})$ : $\mathrm{x}$ produz $(\mathrm{y})$ no grupo $\mathrm{k}\}$. Dessa forma, definem $\mathrm{T}^{\mathrm{m}}=\left\{T^{1} \cup T^{2} \cup T^{3} \cup \cdots \cup T^{k}\right\}$. A Figura 2 fornece uma visualização bidimensional do procedimento.

Figura 2 - Metafronteira e fronteiras dos subgrupos

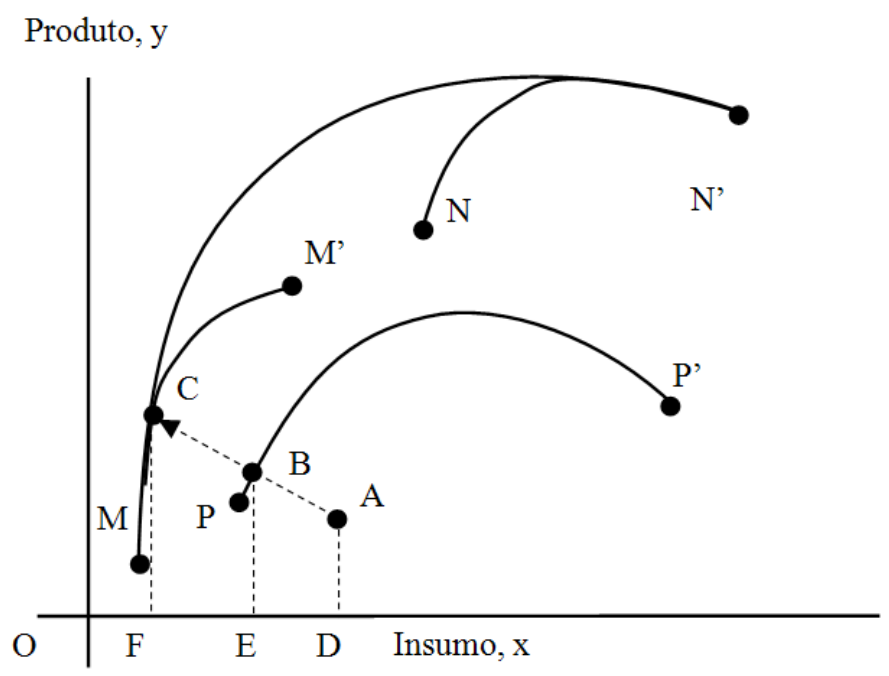

Fonte: Elaboração própria a partir de Chiu et al. (2012).

Nesse processo, extraem-se informações relacionadas à eficiência tecnológica (meta-technology - MTE) e gerencial (group technological efficiency - GTE) 
dos municípios. Formalmente, são $M T E=\theta^{m}$ e $G T E=\theta^{k}$. Os termos $0<\theta^{m} \leq 1$ e $0<\theta^{k} \leq 1$ são escores de eficiência técnica obtidos nas fronteiras MTE e GTE, respectivamente, que representam o potencial de redução dos recursos financeiros da saúde, mantendo fixa a quantidade de produtos e serviços oferecida pelo município. Uma medida de importância da heterogeneidade tecnológica amostral pode ser obtida pela divisão MTR (meta-technology ratio) = MTE/GTE. A abordagem meta-frontier pode representar diferentes estruturas produtivas no setor de saúde, sendo por isso mais adequada nesse caso.

Além disso, uma importante característica dessa amostra são as diferenças de retornos de escala na produção de serviços de saúde. Para municípios menores, há sempre a possibilidade de que a elevação dos gastos aumente mais que proporcionalmente a produção dos serviços em saúde, incorrendo nos chamados retornos crescentes de escala. Para outros municípios, a expansão dos gastos nem sempre produz resultados proporcionais, o que é característica dos retornos decrescentes de escala. Há ainda o caso em que a quantidade produzida é exatamente proporcional ao aumento dos gastos (retornos constantes).

Portanto, mesmo estruturas homogêneas podem apresentar diferenças na resposta produtiva para dado dispêndio. Na modelagem DEA meta-frontier, a curvatura da FPP pode ser flexibilizada para permitir que esse fato seja levado em consideração, através da construção de modelos com retornos variáveis (variable returns to scale - VRS) (BANKER; CHARNES; COOPER, 1984). O procedimento adotado nesta pesquisa possibilita a divisão do indicador de eficiência em dois componentes: a eficiência de escala e a técnica (gerencial). Enquanto o primeiro indica o retorno ótimo de escala, o segundo representa o desempenho municipal na gestão dos recursos, indicador importante para os propósitos deste trabalho. Formalmente, o problema de programação linear (PPL) de um município particular o pode ser representado pelas expressões 2 e 3:

\section{$\max \theta^{m}$}

$$
\begin{aligned}
& \text { s.t. } \sum_{k=1}^{K} \sum_{n=1}^{N_{k}} \lambda_{n}^{k} x_{i n}^{k} \leq x_{i o}^{k}, \quad \quad \mathrm{i}=1,2, \ldots, \mathrm{I} \text {-ésimo insumo } \\
& \sum_{k=1}^{K} \sum_{n=1}^{N_{k}} \lambda_{n}^{k} y_{d n}^{k} \geq \theta^{m} y_{d o}^{k}, \quad \mathrm{~d}=1,2, \ldots, \text { D-ésimo produto } \\
& \sum_{k=1}^{K} \sum_{n=1}^{N_{k}} \lambda_{n}^{k}=1, \quad \mathrm{k}=1 \text { (meta-fronteira) } \\
& \lambda_{n}^{k} \geq 0, \quad \mathrm{n}=1,2, \ldots, \mathrm{N} \text {-ésimo município }
\end{aligned}
$$


$\max \theta^{k}$

$$
\begin{aligned}
& \text { s.t. } \sum_{k=1}^{K} \sum_{n=1}^{N_{k}} \mu_{n}^{k} x_{i n}^{k} \leq x_{i o}^{k}, \quad \mathrm{i}=1,2, \ldots, \text { I-ésimo insumo } \\
& \sum_{k=1}^{K} \sum_{n=1}^{N_{k}} \mu_{n}^{k} y_{d n}^{k} \geq \theta^{k} y_{d o,}^{k} \quad \mathrm{~d}=1,2, \ldots, \text { D-ésimo produto } \\
& \sum_{k=1}^{K} \sum_{n=1}^{N_{k}} \mu_{n}^{k}=1, \quad \mathrm{k}=1,2, \ldots, \mathrm{K} \text {-ésimo subgrupo } \\
& \lambda_{n}^{k} \geq 0, \quad \mathrm{n}=1,2, \ldots, \mathrm{N} \text {-ésimo município }
\end{aligned}
$$

em que $x_{i o}^{k}$ é o vetor de insumos do município o; $x_{i n}^{k}$ são os vetores de insumos dos demais municípios; $y_{d o}^{k}$ é o vetor de produtos do município o; $y_{d n}^{k}$ são os vetores de produtos dos demais municípios; $\lambda_{n}^{k}$ e $\mu_{n}^{k}$ são as variáveis de decisão dos modelos 2 e 3 que projetam o município o para a FPP quando $\theta^{m} \neq 1$ e $\theta^{k} \neq 1$, respectivamente.

Outro aspecto relevante em abordagens não paramétricas é a sensibilidade dos escores de eficiência em relação à presença de observações discrepantes. Por isso, aplicou-se o procedimento sugerido por Sousa e Stošić (2005) para detecção de outliers. Basicamente, os autores propõem uma medida semiparamétrica de dois estágios, baseada nos procedimentos jackknife e bootstrap, para capturar a influência individual sobre toda a amostra. Na primeira fase, o algoritmo estima $(k=$ $1,2, \ldots, K)$ fronteiras excluindo parcialmente uma DMU $n$ para, em seguida, avaliar se ocorreram mudanças significativas nos demais indicadores sem sua presença. Após essa etapa, criam-se regras de comparação sobre os resultados obtidos no primeiro estágio por bootstrap.

Sousa e Stošić (2005) denominam essa medida de leverage, um indicador que varia entre $[0,1]$. Os valores próximos à unidade para uma DMU $n$ sugerem que a sua remoção causa alterações relevantes nos demais escores de eficiência dentro da amostra. O procedimento padrão nesse caso é sua exclusão, pois trata-se de um outlier. De outra forma, valores próximos a zero indicam que a observação não influencia as demais. Como threshold, os autores utilizam 0,02. Assim, para indicadores leverage superiores a 0,02, deve-se excluir a observação da análise. 


\subsection{Análise Exploratória de Dados Espaciais na Eficiência dos Gastos em Saúde}

Para se averiguar a possível relação espacial entre os indicadores de eficiência, utilizou-se a Aede. Conceitualmente, Aede é um conjunto de técnicas capazes de identificar a existência de padrões espaciais (clusters espaciais) (ALMEIDA, 2012). Inicialmente, verifica-se a hipótese de dependência espacial sob a forma de autocorrelação espacial. ${ }^{2}$ Aplica-se, para isso, a estatística I de Moran para se mensurar o grau de associação linear entre a eficiência observada em determinado município no tempo $\left(\theta_{i}^{k}\right)$ e a média ponderada dos valores das regiões vizinhas (defasagens espaciais), denotada por $\left(\theta_{z}^{k}\right)$. Formalmente,

$$
\mathrm{I}=\frac{n}{S_{0}} \frac{\sum_{i} \sum_{z} w_{i z}\left(\theta_{i}^{k}\right)\left(\theta_{z}^{k}\right)}{\sum_{i=1}^{n}\left(\theta_{i}^{k}\right)^{2}},
$$

em que $n$ é o número de regiões; $w_{i z}$ é uma matriz de pesos espaciais que relaciona as regiões i e $z$; e $\mathrm{S}_{0}$ define que todos os elementos da matriz devem ser somados. Neste estudo, adotou-se a matriz do tipo rainha. ${ }^{3}$

A estatística envolvida na estimação do I de Moran tem como hipótese nula a aleatoriedade espacial. Os valores estimados superiores ao esperado $E=-[1 /(n-1)]$ indicam a existência de autocorrelação espacial positiva, isto é, a prevalência de similaridade global entre os valores da eficiência em um município e sua localização espacial. Os valores inferiores, por sua vez, apontam dissimilaridade. Já os indicadores de dependência espacial próximos ao valor esperado sugerem a não rejeição da hipótese nula.

A apresentação dos resultados do I de Moran pode ser feita por diagrama de dispersão, considerando-se a defasagem espacial no eixo vertical e o valor de referência no eixo horizontal. Em resumo, I de Moran pode ser entendido como o coeficiente angular da reta de regressão estimada na função da eficiência contra a defasagem espacial (ANSELIN, 1988).

Para se ter uma medida local de dependência espacial, calcula-se, em adição, o I de Moran local, que faz parte de um conjunto de estatísticas denominadas local indicators of spatial association (Lisa). Esse indicador possibilita a identifi-

2 A autocorrelação espacial mede o quanto a eficiência de um município é afetada por vizinhos.

3 A matriz rainha classifica como vizinhas regiões que compartilham fronteiras geográficas físicas e os vértices do mapa. Por sua ampla definição de vizinhos, LeSage (1999) recomenda seu uso em estudos empíricos. Ademais, aplicando-se o procedimento de Baumont, Ertur e Gallo (2004), testaram-se outras seis matrizes de pesos espaciais (torre e $k$-vizinhos - de 1 a 5), e não foram observadas alterações expressivas nos indicadores de autocorrelação espacial. 
cação de clusters espaciais estatisticamente significativos para cada observação, assumindo a propriedade de que a soma dos indicadores locais seja proporcional ao indicador global correspondente. O I de Moran local pode ser expresso por:

$$
I_{i}=\left(\theta_{i}^{k}\right) \sum_{z=1}^{n} w_{i z}\left(\theta_{z}^{k}\right)
$$

Através do indicador local, é possível identificar quatro tipos de associações espaciais: alto-alto (AA), alto-baixo (AB), baixo-alto (BA) e baixo-baixo (BB). ${ }^{4} \mathrm{Um}$ agrupamento do tipo $\mathrm{AA}$, por exemplo, indica que um município de alta eficiência está associado espacialmente a vizinhos com melhor gestão em saúde. Um agrupamento BB, por sua vez, indica que um município ineficiente está associado espacialmente a outros que também apresentam baixa eficiência, o que pode indicar sérios problemas de desperdício de recursos em âmbito regional. As demais interpretações podem ser feitas de forma análoga.

\subsection{Descrição das Variáveis e Fonte de Dados}

Esta pesquisa analisou os 853 municípios do estado Minas Gerais, em 2010, ano do último censo divulgado e mais recente com disponibilidade completa de informações. As variáveis inputs e outputs foram selecionadas após uma revisão dos estudos empíricos em saúde, considerando-se a disponibilidade no presente contexto (FARIA; JANNUZZI; SILVA, 2008; HADAD; HADAD; SIMON-TUVAL, 2013; ROCHA et al., 2017). Para representar os insumos municipais, foi utilizado o gasto per capita em saúde (gastopc). Essa variável soma os gastos orçamentários anuais correntes em 2010, considerando os recursos destinados à atenção básica, assistência hospitalar e ambulatorial, suporte profilático e terapêutico, vigilância sanitária, epidemiológica e alimentação/nutrição (FUNDAÇÃO JOÃO PINHEIRO, 2016).

O conjunto de produtos incluiu: a cobertura vacinal tetravalente em menores de um ano (tetra), que considera a proporção da referida população vacinada contra difteria, coqueluche, tétano e haemophilus influenza tipo b; a cobertura vacinal contra poliomielite em menores de um ano (poli); a cobertura vacinal contra febre amarela em menores de um ano (febre); a cobertura vacinal contra influenza da população residente com 60 anos ou mais (influ); a cobertura vacinal contra hepatite em menores de 20 anos de idade (hep); e a cobertura vacinal de tríplice viral na população de um ano de idade (viral) (DATASUS, 2016).

$4 \quad$ Para mais detalhes, ver LeSage (1999). 
Além das coberturas vacinais, utilizou-se o índice de desenvolvimento humano - longevidade (IDHL), um dos três indicadores que compõem o índice de desenvolvimento humano municipal (IDHM). A variável é construída a partir de informações sobre a expectativa de vida ao nascer, considerando-se o padrão de mortalidade prevalecente no ano do censo.

\section{Resultados e Discussões}

Dado o problema da influência de observações discrepantes na construção de fronteiras não paramétricas, realizou-se o procedimento de detecção proposto por Sousa e Stošić (2005) na amostra global. Não houve evidências de outliers significativos, pois os leverages calculados se situaram abaixo do limite máximo estabelecido por Sousa e Stošić (2005), que é de 0,02. A Figura 3 apresenta a frequência do teste leverage, realizado sobre a metafronteira.

Figura 3 - Frequência dos leverages da eficiência dos gastos em saúde

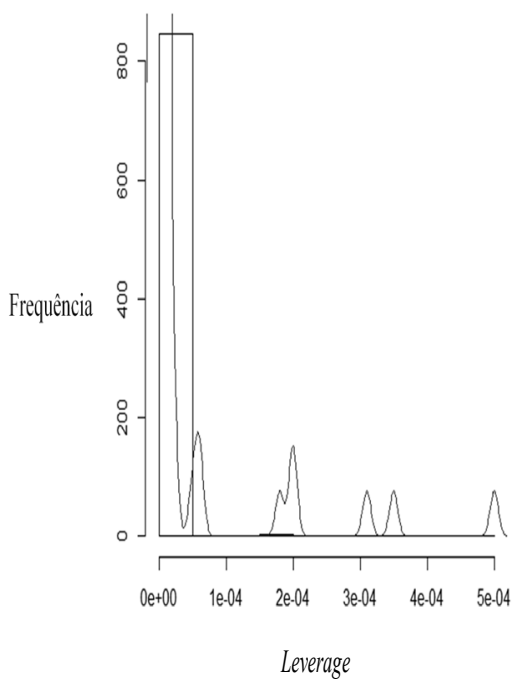

Fonte: Elaboração própria.

Após esse procedimento, construíram-se fronteiras locais para controlar parte da heterogeneidade da amostra, dividindo-a previamente por critério populacional. Os municípios foram separados em três grupos: a) de até 50 mil habitantes (92,02\% do total de municípios da amostra); b) com a partir de 50 mil até 100 mil habitantes (4,33\%); e c) acima de 100 mil habitantes (3,63\%).

A estimação feita dentro das fronteiras retrata o desempenho relativo dos municípios quando comparados com outras unidades que enfrentam problemas 
e limitações semelhantes. A distância de um determinado município da fronteira global, por sua vez, mostra a relevância do fator populacional na determinação da eficiência. A Tabela 1 resume os principais resultados do DEA por grupos. Na parte $a$, foram inseridas estatísticas descritivas dos indicadores de eficiência. Na parte $b$, incluíram-se informações sobre a média do gasto público per capita, da cobertura vacinal e do IDHL.

Tabela 1 - Estatísticas descritivas dos indicadores de eficiência

\begin{tabular}{|c|c|c|c|c|}
\hline \multicolumn{5}{|c|}{ Parte a: indicadores de eficiência por grupos } \\
\hline Grupos & Quantidade & Média & Mínimo & Máximo \\
\hline \multicolumn{5}{|l|}{ Taxa metatecnologia (MTR) } \\
\hline Até 50 mil habitantes & 785 & 0,970 & 0,454 & 1,00 \\
\hline De 50 a 100 mil habitantes & 37 & 0,633 & 0,300 & 1,00 \\
\hline A partir de 100 mil habitantes & 31 & 0,690 & 0,366 & 1,00 \\
\hline \multicolumn{5}{|c|}{ Eficiência na metafronteira (MTE) } \\
\hline Até 50 mil habitantes & 785 & 0,413 & 0,098 & 1,00 \\
\hline De 50 a 100 mil habitantes & 37 & 0,429 & 0,117 & 1,00 \\
\hline A partir de 100 mil habitantes & 31 & 0,463 & 0,122 & 1,00 \\
\hline \multicolumn{5}{|c|}{ Eficiência dentro do grupo (GTE) } \\
\hline Até 50 mil habitantes & 785 & 0,429 & 0,107 & 1,00 \\
\hline De 50 a 100 mil habitantes & 37 & 0,667 & 0,251 & 1,00 \\
\hline A partir de 100 mil habitantes & 31 & 0,664 & 0,168 & 1,00 \\
\hline \multicolumn{5}{|c|}{ Parte b: médias das variáveis da fronteira por grupos } \\
\hline Grupos & Quantidade & Gastopc & Cobvac & IDHL \\
\hline Até 50 mil habitantes & 785 & $\mathrm{R} \$ 375$ & $93 \%$ & 0,821 \\
\hline De 50 a 100 mil habitantes & 37 & $\mathrm{R} \$ 337$ & $91 \%$ & 0,851 \\
\hline A partir de 100 mil habitantes & 31 & $\mathrm{R} \$ 346$ & $91 \%$ & 0,854 \\
\hline
\end{tabular}

Fonte: Elaboração própria.

As razões metatecnologia (MTR) representam a média da divisão entre os escores obtidos dentro do grupo (GTE) e da metafronteira (MTE). Quando não existem discrepâncias provocadas pelo tamanho da população, os indicadores GTE e MTE se igualam e a MTR torna-se unitária. Na medida em que GTE e MTE se distanciam, MTR tende para zero. Dessa forma, os resultados reportados na Tabela 1 indicam que municípios de médio (MTR $=0,633)$ e grande $(\mathrm{MTR}=0,690)$ portes são mais impactados pela influência tecnológica na análise DEA. Pode-se notar que a eficiência dessas regiões foi subestimada quando o fator tamanho não foi considerado. Os contrastes verificados entre indicadores MTE e GTE reforçam tal constatação. 
A eficiência média dentro dos grupos (a qual leva em consideração a heterogeneidade) foi de 0,429, 0,667 e 0,664 nos municípios de pequeno, médio e grande portes, respectivamente. Dessa forma, é possível dizer que as pequenas regiões são, em média, mais ineficientes na gestão dos gastos públicos em saúde. Medici (2011) explica que regiões de pequeno porte populacional não têm, em geral, acesso ágil e integral aos atendimentos de saúde necessários. Para suprir parte da demanda são investidos recursos no sistema de saúde na forma de pequenos estabelecimentos, os quais normalmente funcionam com alto custo fixo médio. Por esse motivo, entre outros, municípios pequenos tendem a ser mais ineficientes, visto que não há escala suficiente para ofertar diversos atendimentos em saúde. Assim, os investimentos realizados elevam o gasto per capita em comparação aos de maior porte (percebe-se que o gasto per capita médio desse grupo foi superior aos demais - Tabela 1, parte b).

Para se ter uma melhor descrição dos dados, são apresentadas, na Tabela 2, médias de alguns indicadores por faixas de eficiência.

Tabela 2 - Médias de indicadores por faixas de eficiência (2010)

\begin{tabular}{lcccc}
\hline Variáveis & De 0,00 até $\mathbf{0 , 2 5}$ & De 0,25 até 0,50 & De 0,50 até 0,75 & Mais de 0,75 \\
\hline Gastopc & 557,28 & 367,82 & 385,05 & 148,77 \\
Cobvac & 90,75 & 93,12 & 94,91 & 95,76 \\
IDHL & 0,821 & 0,818 & 0,820 & 0,862 \\
Pop & $33.630,17$ & 4.470 & $18.576,50$ & $69.337,50$ \\
\hline
\end{tabular}

Fonte: Elaboração própria.

Nota: gastopc $=$ gasto per capita em saúde $(\mathrm{R} \$)$; cobvac = cobertura vacinal média (\%); $\mathrm{IDHL}=$ índice de desenvolvimento humano - longevidade (varia entre zero e um); pop = população (pessoas).

Em termos médios, um dos problemas dos municípios na faixa mais baixa de eficiência são os elevados gastos per capita ( $\mathrm{R} \$ 557,28)$, visto que os demais fatores foram próximos, em valores, dos observados em outras faixas. Por exemplo, a cobertura vacinal média desses municípios, apesar de ser menor em relação aos demais, mostrou-se superior a 90\%. O IDHL também se situou próximo da média dos demais. Por seu turno, os municípios da faixa de eficiência mais elevada obtiveram indicadores médios de cobertura vacinal e longevidade (IDHL) acima daqueles da primeira faixa (os menos eficientes), investindo $73 \%$ a menos no setor (per capita). Nota-se que a média populacional dessas regiões também foi maior em comparação às outras faixas.

Para se obter a dimensão da correlação espacial da eficiência dos gastos em saúde, estimou-se o I de Moran global. A associação entre os indicadores de eficiência (eixo horizontal) e a defasagem espacial (eixo vertical) é mostrada na Figura 4. 
Figura 4 - Gráfico da regressão entre eficiência e defasagem espacial

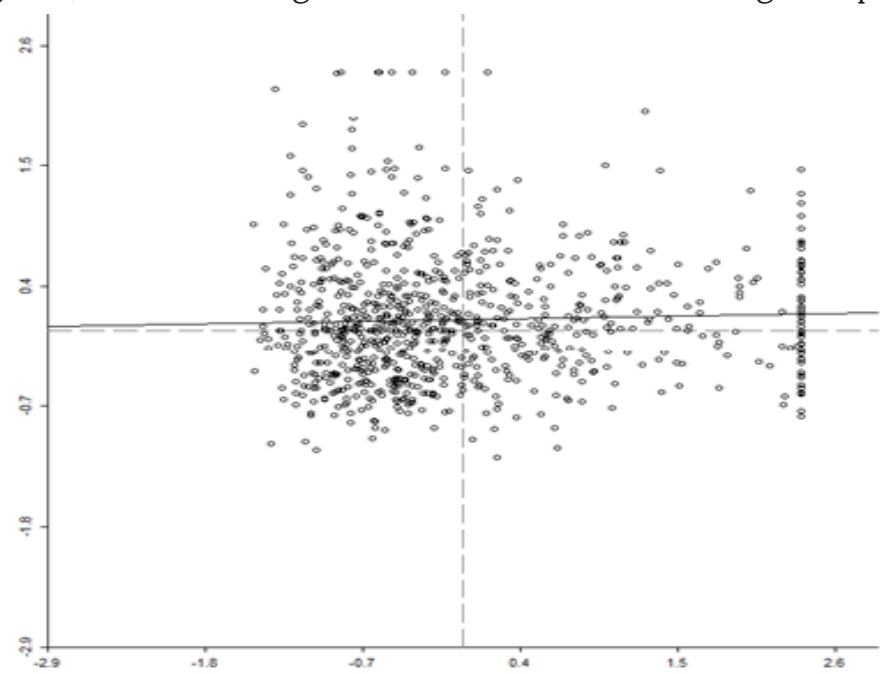

I de Moran = 0,021

Fonte: Elaboração própria.

A inclinação da reta (I de Moran global) foi de 0,021, porém não significativa. Isto é, os indicadores de eficiência não estão espacialmente correlacionados. Conforme a Figura 4, a distribuição está concentrada no centro, em forma de "nuvem". A falta de padrão global sugere que não existe efeito aprendizagem significativo em Minas Gerais. Por outro lado, a estimativa do I de Moran local revelou alguns casos positivos (e outros negativos) de agrupamentos de municípios com alta eficiência (AA) (e baixa eficiência, BB). A Figura 5 apresenta visualmente esses clusters de eficiência em Minas Gerais. 
Figura 5 - Clusters de eficiência dos municípios de Minas Gerais (2010)

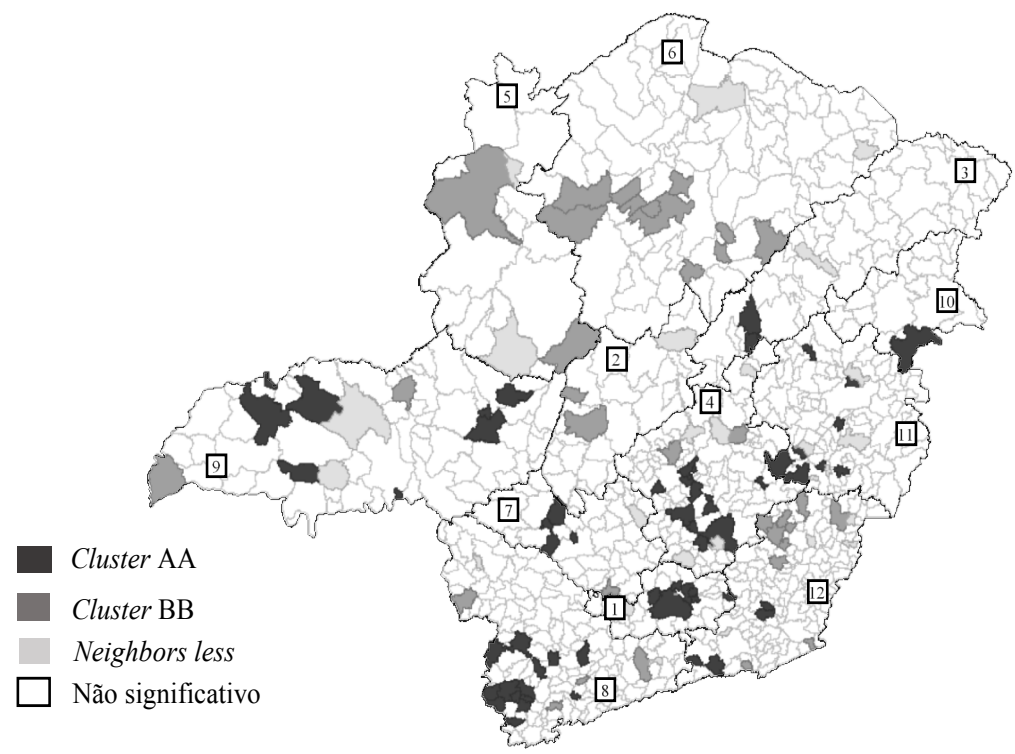

Fonte: Elaboração própria.

Nota: As mesorregiões de Minas Gerais foram identificadas do seguinte modo: 1) Campo das Vertentes; 2) Central Mineira; 3) Vale do Jequitinhonha; 4) Região Metropolitana de Belo Horizonte; 5) Noroeste; 6) Norte; 7) Oeste; 8) Sul e Sudoeste; 9) Triângulo Mineiro e Alto Paranaíba; 10) Vale do Mucuri; 11) Vale do Rio Doce; e 12) Zona da Mata.

Entre as formações de clusters, deu-se atenção aos agrupamentos AA no Sul, região Metropolitana de Belo Horizonte, Triângulo Mineiro e Alto Paranaíba, Campo das Vertentes e Vale do Rio Doce. Isto é, são municípios de alta eficiência que estão rodeados por outros com padrão semelhante. Analisar as particularidades dessas regiões é caminho natural para melhorar a gestão dos recursos nas demais regiões do estado. Por outro lado, destacaram-se os agrupamentos de municípios $\mathrm{BB}$ das regiões Noroeste, Norte e Zona da Mata. O conhecimento acerca da realidade desses municípios com baixa eficiência também pode servir de aprendizado. Com esse intuito, apresentam-se, na Tabela 5 , indicadores de eficiência e variáveis socioeconômicas de alguns municípios AA e BB. 


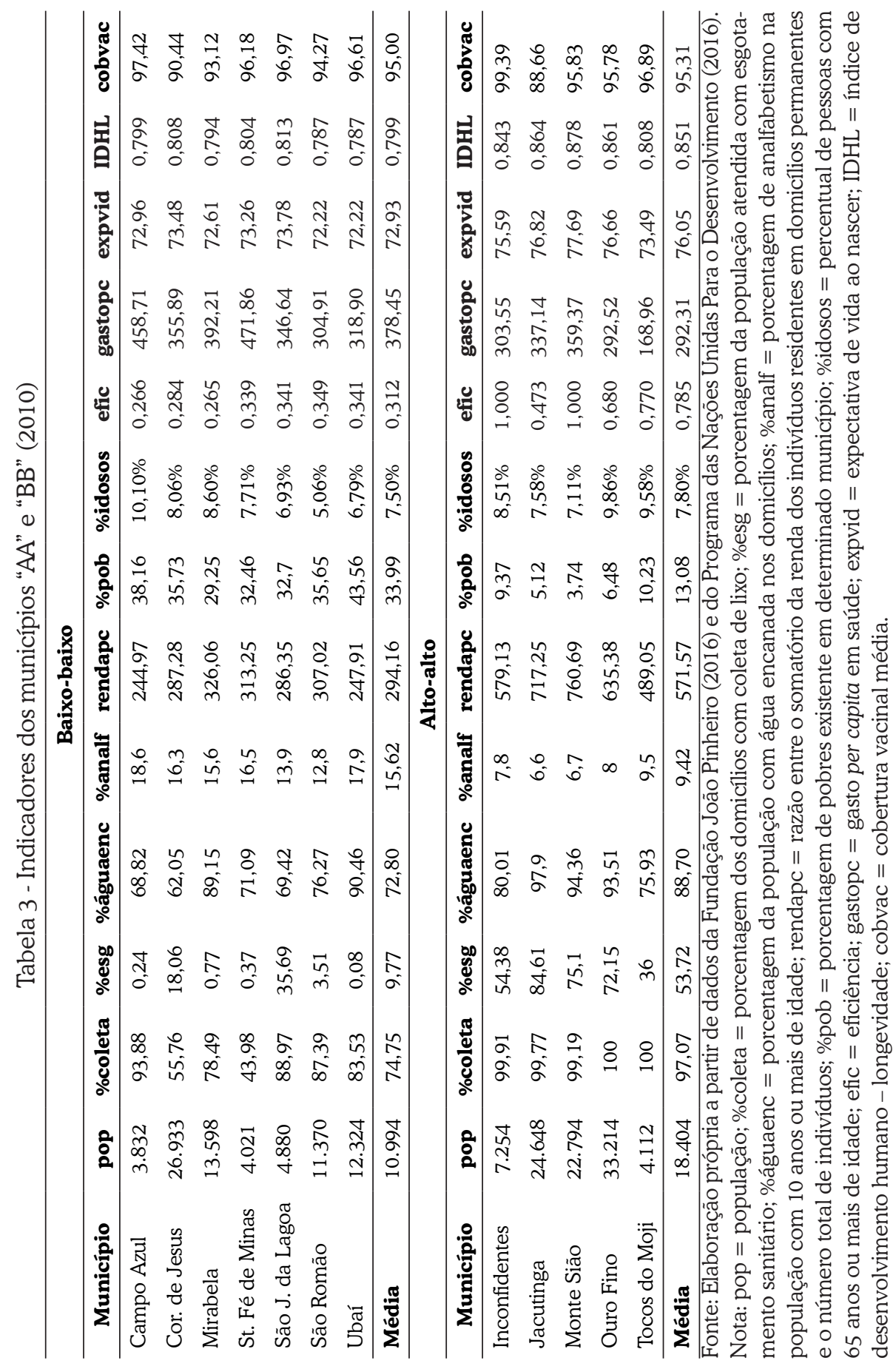


As regiões do cluster $\mathrm{BB}$, localizadas no Norte do estado, possuem indicadores de cobertura vacinal acima de 90\% (em média, 95\%). No entanto, notam-se baixos valores nos indicadores de qualidade de vida (expectativa de vida e IDHL). Esse problema é reflexo, principalmente, dos gargalos de oferta dos serviços de saúde, os quais estão diretamente relacionados com a qualidade de vida da população. Além disso, por questões já discutidas nesta seção, o alto gasto per capita (comparado ao grupo AA) pode estar associado ao elevado custo fixo médio. A redução dos gastos per capita possível para esses municípios, considerando-se o mínimo estabelecido por lei, poderia ser de $20 \%$ em média.

A fim de se analisar mais detalhadamente os clusters, foram acrescentadas à Tabela 3 indicadores de coleta de lixo, esgotamento sanitário, renda, escolaridade, situação da água encanada, população idosa e de baixa renda desses municípios. Essas informações foram extraídas da base de dados eletrônica Índice Mineiro de Responsabilidade Social, da Fundação João Pinheiro (FJP, 2016), para o ano de 2010.

De forma geral, é possível perceber que os indicadores socioeconômicos do grupo AA são mais elevados que os do grupo BB, em média. Percebe-se que, nas cidades menos eficientes, um a cada quatro domicílios não possui coleta de lixo, enquanto quase a totalidade tem acesso nas regiões eficientes. De forma análoga, o percentual dos domicílios com esgotamento sanitário e água encanada variou drasticamente em favor dos mais eficientes. Esses dados, apesar de simples, servem para indicar possíveis entraves do sistema de saúde nesses municípios, já que a situação sanitária interfere diretamente na condição e bem-estar da população.

Notou-se também que as regiões AA apresentaram maior renda, menor proporção de pessoas pobres e indicadores de educação mais elevados. A importância desses atributos na determinação da eficiência em saúde é documentada em diversos estudos. Daniel e Gomes (2015) mostram que municípios de maior renda per capita são mais eficientes na gestão dos recursos em saúde. Nesse mesmo estudo, a pobreza relacionou-se negativamente ao indicador. Constatações semelhantes são verificadas por Hadad, Hadad e Simon-Tuval (2013). Buss e Pellegrini Filho (2007) argumentam que a baixa renda e escolaridade refletem-se na falta de oportunidades de acesso à saúde.

Sobre a educação, Daniel e Gomes (2015) relatam, ainda, que o estoque de capital humano, mensurado pela escolarização, tende a elevar as exigências da população sobre a qualidade dos serviços prestados e a aplicação dos recursos. Esse movimento cria uma espécie de ciclo virtuoso (ou vicioso) no contexto da saúde, uma vez que a elevação da renda e educação pode caminhar em direção à otimização dos recursos em saúde, que, por sua vez, permite novamente sua expansão, pois o acesso e a qualidade dos serviços prestados são condições necessárias para melhorar os indicadores de desenvolvimento municipal. Assim, para 
elevar os indicadores de eficiência municipal em saúde, os gestores públicos deveriam considerar aspectos além dos gastos no setor, como a integração regional do sistema e as condições sanitárias e socioeconômicas das regiões.

\section{Considerações Finais}

Este trabalho buscou se inserir na literatura especializada sobre gestão em saúde, fornecendo uma análise mais profunda da questão espacial da eficiência dos gastos públicos no setor. Especificamente, buscou-se construir e relacionar espacialmente o indicador de eficiência dos municípios de Minas Gerais em 2010. Para tanto, os métodos DEA e Aede foram adotados.

Cabe ressaltar, em primeiro lugar, que a metodologia DEA meta-frontier mostrou-se mais adequada para se analisar a eficiência municipal em saúde. Isso porque os resultados exibiram grande divergência quando a heterogeneidade amostral foi considerada, sobretudo nas amostras de municípios de médio e grande portes. Nesse sentido, este estudo avança em relação aos demais trabalhos da área aplicados ao estado ao considerar explicitamente esse fato.

Por outro lado, os resultados gerais apresentados dialogam com os trabalhos anteriores, indicando baixa eficiência na alocação dos recursos. A literatura vem apontando os problemas no acesso aos serviços (como número insuficiente de profissionais, tempo de espera para a realização de exames e atendimentos, falta de vagas para internações, dificuldades de locomoção intermunicipal, etc.) como os principais fatores responsáveis por esse quadro.

Em pareamento com os trabalhos anteriores, os resultados desta pesquisa indicam gastos per capita mais elevados nos municípios ineficientes e pequenos em relação aos mais eficientes. Ocorre que municípios menores usualmente ofertam serviços de saúde com elevados custos fixos médio e sua população tem mais dificuldades em usufruir de procedimentos de média e alta complexidades por uma questão de distanciamento. Dessa forma, um desafio dos gestores públicos é diminuir esses problemas de acesso diante de dificuldades orçamentárias, estruturais e até de fixação de recursos humanos em regiões mais afastadas dos grandes centros.

Por fim, a presente pesquisa também traz o apontamento de que as condições socioeconômicas e sanitárias, entre outras, definidas ex ante à situação de saúde podem ser entraves para a melhoria dos indicadores de eficiência do gasto em saúde. Por exemplo, se determinado município não oferece saneamento básico (coleta e tratamento de lixo, esgotamento sanitário ou água potável), a procura por serviços de saúde pode aumentar em razão do possível surgimento de doenças correlatas. Dessa forma, os gestores públicos deveriam considerar tais informações na formulação de políticas públicas. 


\section{Referências}

ALMEIDA, E. Econometria espacial aplicada. São Paulo: Alinea, 2012.

ANSELIN, L. Spatial econometrics: methods and models. [S.l.]: Kluwer Academic Publishers, 1988.

BANKER, R. D.; CHARNES, A.; COOPER, W. W. Some models for estimating technical and scale inefficiencies in data envelopment analysis. Management Science, v. 30, n. 9, p. 10781092, Sept. 1984. Disponível em: <http://www.utdallas.edu/ ryoung/phdseminar/BCC1984. pdf >. Acesso em: 13 nov. 2016.

BAUMONT, C.; ERTUR, C.; GALLO, J. Spatial analysis of employment and population density: the case of the agglomeration of Dijon 1999. Geographical Analysis, v. 36, n. 2, p. 146-176, July 2004. Disponível em: <https://onlinelibrary.wiley.com/doi/abs/10.1111/j.1538-4632.2004. tb01130.x>. Acesso em: 13 nov. 2016.

BRASIL. Constituição da República Federativa do Brasil de 1988. Brasília, 1988. Disponível em: <http://www.planalto.gov.br/ccivil_03/Constituicao/Constituicao.htm>. Acesso em: 13 nov. 2016.

BUSS, P. M.; PELLEGRINI FILHO, A. P. A saúde e seus determinantes sociais. Physis: Revista de Saúde Coletiva, Rio de Janeiro, v. 17, n. 1, p. 77-93, 2007. Disponível em: <http://www. scielo.br/pdf/physis/v17n1/v17n1a06.pdf>. Acesso em: 13 nov. 2016.

CHIU, C. R. et al. Decomposition of the environmental inefficiency of the meta-frontier with undesirable output. Energy Economics, v. 34, n. 5, p. 1392-1399, Sept. 2012. Disponível em: < https://www.sciencedirect.com/science/article/pii/S0140988312001089>. Acesso em: 13 nov. 2016.

DANIEL, L. P.; GOMES, A. P. Eficiência na oferta de serviços públicos de saúde nos municípios do estado de Mato Grosso. Reflexões Econômicas, Ilhéus, BA, v. 1, n. 1, p. 179-218, abr./set. 2015. Disponível em: < http://periodicos.uesc.br/index.php/reflexoeseconomicas/ article/view/828 > . Acesso em: 13 nov. 2016.

DATASUS. Departamento de Informática do SUS. 2016. Disponível em: < http://www2. datasus.gov.br/DATASUS/index.php?area=01 >. Acesso em: 13 nov. 2016.

FÄRE, R. et al. Characteristics of a polluting technology: theory and practice. Journal of Econometrics, v. 126, n. 2, p. 469-492, June 2005. Disponível em: < https://www.sciencedirect. com/science/article/pii/S0304407604001204>. Acesso em: 13 nov. 2016.

FARIA, F. P.; JANNUZZI, P. M.; SILVA, S. J. Eficiência dos gastos municipais em saúde e educação: uma investigação através da análise envoltória no estado do Rio de Janeiro. Revista de Administração Pública, Rio de Janeiro, v. 42, n. 1, p. 155-177, jan./fev. 2008. Disponível em: <http://www.scielo.br/pdf/rap/v42n1/a08v42n1.pdf>. Acesso em: 13 nov. 2016.

FELDER, S.; TAUCHMANN, H. Federal state differentials in the efficiency of health production in Germany: an artifact of spatial dependence? The European Journal of Health Economics, v. 14, n. 1, p. 21-39, Feb. 2013. Disponível em: <https://link.springer.com/ article/10.1007/s10198-011-0345-8>. Acesso em: 13 nov. 2016. 
FERREIRA, M. P.; PITTA, M. T. Avaliação da eficiência técnica na utilização dos recursos do sistema único. São Paulo em Perspectiva, São Paulo, v. 22, n. 2, p. 55-71, jul./dez. 2008. Disponível em: <http://produtos.seade.gov.br/produtos/spp/v22n02/v22n02_05.pdf>. Acesso em: 13 nov. 2016.

FONSECA, P. C.; FERREIRA, M. A. M. Investigação dos níveis de eficiência na utilização de recursos no setor de saúde: uma análise das microrregiões de Minas Gerais. Saúde e Sociedade, São Paulo, v. 18, n. 2, p. 199-213, 2009. Disponível em: < http://www.scielo.br/ pdf/sausoc/v18n2/04.pdf>. Acesso em: 13 nov. 2016.

FUNDAÇÃO JOÃO PINHEIRO. Índice Mineiro de Responsabilidade Social. Belo Horizonte, 2016. Disponível em: < http://imrs.jjp.mg.gov.br>. Acesso em: 13 jul. 2016.

HADAD, S.; HADAD, Y.; SIMON-TUVAL, T. Determinants of healthcare system's efficiency in OECD countries. The European Journal of Health Economics, v. 14, n. 2, p. 253-265, Apr. 2013. Disponível em: <https://link.springer.com/article/10.1007/s10198-011-0366-3>. Acesso em: 13 nov. 2016.

KOOPMANS, T. Activity analysis of production and allocation. New York: John Wiley $\mathcal{3}$ Sons, 1951.

LESAGE, J. P. The theory and practice of spatial econometrics. [S. 1.], 1999. Disponível em: <https://www.spatial-econometrics.com/html/sbook.pdf>. Acesso em: 30 nov. 2016.

MACHADO JÚNIOR, S. P.; IRFFI, G.; BENEGAS, M. Análise da eficiência técnica dos gastos com educação, saúde e assistência social dos municípios cearenses. Planejamento e Políticas Públicas, Brasília, n. 36, p. 87-113, jan./jun. 2011. Disponível em: <http://www.ipea.gov.br/ ppp/index.php/PPP/article/view/223/204>. Acesso em: 30 nov. 2016.

MARQUES, A. J. S. et al (Org.). O choque de gestão na saúde em Minas Gerais. Belo Horizonte: Secretaria de Estado de Saúde de Minas Gerais, 2009. Disponível em: < http://bvsms.saude. gov.br/bvs/publicacoes/choque_gestao_saude_minas_gerais.pdf > . Acesso em: 30 nov. 2016.

MAZON, L. M.; MASCARENHAS, L. P. G.; DALLABRIDA, V. R. Eficiência dos gastos públicos em saúde: desafio para municípios de Santa Catarina, Brasil. Saúde e Sociedade, São Paulo, v. 24, n. 1, p. 23-33, 2015. Disponível em: < http://www.scielo.br/pdf/sausoc/v24n1/01041290-sausoc-24-1-0023.pdf>. Acesso em: 30 nov. 2016.

MEDICI, A. Propostas para melhorar a cobertura, a eficiência e a qualidade no setor saúde. In: BACHA, E. L.; SCHWARTZMAN, S. (Org.). Brasil: a nova agenda social. Rio de Janeiro: LTC, 2011. p. 23-93.

O'DONNELL, C.; RAO, D. S. P.; BATTESE, G. E. Metafrontier frameworks for the study of firm-level efficiencies and technology ratios. Empirical Economics, v. 34, n. 2, p. 231-255, Mar. 2008. Disponível em: <https://link.springer.com/article/10.1007/s00181-007-0119-4>. Acesso em: 30 nov. 2016.

PROGRAMA DAS NAÇÕES UNIDAS PARA O DESENVOLVIMENTO. Atlas do Desenvolvimento, 2016. Disponível em: < http://www.atlasbrasil.org.br/2013>. Acesso em: 30 nov. 2016. 
QUEIROZ, M. F. M. et al. Eficiência no gasto público com saúde: uma análise nos municípios do Rio Grande do Norte. Revista Econômica do Nordeste, Fortaleza, v. 44, n. 3, p. 761-776, jul./set. 2013. Disponível em: <https://www.bnb.gov.br/projwebren/Exec/artigoRenPDF. aspx?cd_artigo_ren=1392>. Acesso em: 30 nov. 2017.

ROCHA, F. et al. Are more resources always the answer? A supply and demand analysis for public health services in Brazilian municipalities. EconomiA, v. 18, n. 1, p. 98-116, Jan./Apr. 2017.

SANTERRE, R. E.; NEUN, S. P. Health economics: theory, insights, and industry studies. Stamford: Cengage Learning, 2012.

SHEPHARD, R. W. Theory of cost and production functions. Princeton: Princeton University Press, 1970.

SILVA, A. A. P. et al. Eficiência na alocação de recursos públicos destinados à educação, saúde e habitação em municípios mineiros. Contabilidade, Gestão e Governança, Brasília, DF, v. 15, n. 1, p. 96-114, jan./abr. 2012. Disponível em: <https://cgg-amg.unb.br/index.php/ contabil/article/view/389/pdf > . Acesso em: 30 nov. 2016.

SOUSA, M. C. S.; STOŠIĆ, B. Technical efficiency of the Brazilian municipalities: correcting nonparametric frontier measurements for outliers. Journal of Productivity Analysis, v. 24, n. 2, p. 157-181, Oct. 2005. Disponível em: < https://ink.springer.com/article/10.1007/s11123-0054702-4>. Acesso em: 30 nov. 2016.

WORLD BANK. World development indicators database. Washington, DC, 2016. Disponível em: < https://data.worldbank.org >. Acesso em: 30 nov. 2016.

Recebido em: 24/01/2017. Aceito em: 21/07/2017. 